\title{
Implementation and Comparison of Different Segmentation Techniques for Medical Images
}

\author{
Kishore Gunna, PhD \\ MVR College of Engineering \& \\ Technology, Vijayawada
}

\begin{abstract}
Segmentation is an important concept in image processing with an objective of dividing the image into regions and characterizes the structures with some input features, so that the output image is meaningful and easier to analyze. A large number of algorithms have been proposed in various application areas. In medical field the segmentation plays an important role helping doctors to take appropriate decisions. Identifying the region of interest is an important task in the medical field. Soft tissue of human bodies can be produced using the Magnetic Resonance Images. In this paper brain image segmentation is done using the K-Means, Fuzzy CMeans, Otsu Thresholding and morphological closing and reconstruction. Performance measuring parameters such as Structural content, mean square value, peak to signal ratio, Average difference Results obtained are satisfactory
\end{abstract}

\section{General Terms}

Image Processing, Segmentation,Clustering Techniques

\section{Keywords}

Segmentation, K-Means, Fuzzy C-Means, Otsu Thresholding., PSNR, MSE, Structural Content.

\section{INTRODUCTION}

In Image processing the term "Image segmentation is the process of partitioning a digital image into multiple segments". By using the concept of segmentation we can simplify and change the representation of an image into something that is more meaningful and easier to analyze [1]. Image segmentation had become a major research topic in Image processing as most of the computer vision and image analysis problems require detecting the objects or dividing the image into regions which are considered to be uniform, given by its criteria (color, motion, texture etc).A large number of algorithms are available for segmentation of the digital images, where the process slightly varies from one method to another. In spite of the availability of these large numbers of algorithms we are not able to find an algorithm that can be applied to all type of digital images. An algorithm developed for one group of images may not be applied to another class of images and we may not get the same results.[2,3].

The image segmentation generally can be Discontinues based and similarities based. In discontinuous based the partition of image is based on the abrupt change in the intensity, like edge detection [4]. In the later approach includes thresholding, region growing where the image partition based on a set of predefined criteria[4].Image segmentation has a number of applications. Medical image segmentation is one of the important application in which the paper is focusing. In medicine today we can see that the diagnostic imagining has become a vital tool. The images provide a means for mapping the anatomy of the subject. The technology had played a great role in diagnosis and treatment planning. The use of computers in this field has increased and the image segmentation algorithms playing a vital role in the imagining applications such as the quantification of tissue volumes [5], diagnosis [6],localization of pathology [7], study of anatomical structure [8], treatment planning[9], partial volume correction of functional imaging data [10], and computer integrated surgery $[11,12]$.

The segmentation of an image is influenced by three main characteristics; Object boundaries, object homogeneity and object shape. Object boundaries and object homogeneity are image or signal based characteristics, hence they are affected by Noise or reconstruction artifacts. these are also modality dependent as we can see that computed tomography (CT) image a have dominant boundaries than the ultrasound image. The shape of an object changes from one image to another and in most imaging modalities — apart from small deviations like perspectival mapping distortions - also independent from the acquisition technique. Thus developing the Segmentation methods object boundary, object homogeneity and object shape have a strong influence on them. The segmentation techniques can be divided into four Thresholding method, Clustering Approach ,Region Based Approaches ,Edge Detection Approach[13].A number of algorithms are also developed now a days for the segmentation. There are other methods like Histogram based, Graph partitioning methods and a number of hybrid models are being developed. The methods like thresholding or region growing rely more on the image or signal information. In segmentation process objects shape is important hence many models focus on it. The classification of segmentation approaches done according to the amount of boundary, homogeneity or shape knowledge they incorporate. In literature many surveys have been done on the segmentation of the images. segmentation techniques are categorized into four groups: region-based, boundary-based, hybrid and atlasbased where each group is defined by the image features used by the segmentation technique[14]. The threshold, edge and region-based methods are distinguished in[15]. In [16] a detail segmenatation methods have been discussed which includes Thresholding approaches, region growing methods, classifiers, clustering methods, Markov random field models, artificial neural networks, deformable models and atlas guided methods.

In this paper segmentation of medical images has been studied using fuzzy c-means, k-means, otsu Thresholding and morphological closing and reconstruction

\section{METHODOLOGY}

\subsection{Otsu Method}

Otsu [18]in 1979 has developed Otsu's Thresholding Method based on the simple idea that is to find the threshold that minimizes the weighted within-class variance. He made several assumptions as the Histogram (and the image) are bimodal. He also limited to no use of spatial coherence, nor any other notion of object structure. He assumed the stationary statistics, but can be modified to be locally 
adaptive. And finally assumed uniform illumination(implicitly), so the bimodal brightness behavior arises from object appearance differences only. This method uses discriminate analysis. Pixels of the image are portioned into two classes $\mathrm{C} 0$ and $\mathrm{C} 1$, At a gray level t $\mathrm{C} 0=\{0,1,2, \ldots$, $\mathrm{t}\}$ and $\mathrm{Cl}=\{\mathrm{t}+1, \mathrm{t}+2, \ldots \mathrm{L}-1\}$.we can define the withinclass variance, between-class variance, and the total variance as $\sigma_{2 \mathrm{w}}, \sigma_{2 \mathrm{~B}}$ and $\sigma_{2 \mathrm{~T}}[17]$.

Minimizing the criteria functions with respect to $t$ an optimal threshold is defined [19]

$\lambda=\frac{\sigma_{\mathrm{B}}^{2}}{\sigma_{\mathrm{W}}^{2}}, \eta=\frac{\sigma_{\mathrm{B}}^{2}}{\sigma_{\mathrm{T}}^{2}}, \kappa=\frac{\sigma_{\mathrm{T}}^{2}}{\sigma_{\mathrm{W}}^{2}}$

Here the $\eta$ is considered as it is simple and the optimal threshold is defined as $t=\operatorname{Arg}$ Min $\eta$ where the

$$
\sigma_{\mathrm{T}}^{2}=\sum_{\mathrm{i}=0}^{\mathrm{L}-1}\left[1-\mu_{\mathrm{T}}\right]^{2} \mathrm{P}_{\mathrm{i}}, \mu_{\mathrm{T}}=\sum_{\mathrm{i}=0}^{\mathrm{L}-1}\left[\mathrm{i} \mathrm{P}_{\mathrm{i}}\right]
$$

And $\sigma_{\mathrm{B}}^{2}=\mathrm{W}_{0} \mathrm{~W}_{1}\left(\mu_{0} \mu_{1}\right)^{2}, \mathrm{~W}_{0}=\sum_{\mathrm{i}=0}^{\mathrm{t}} \mathrm{P}_{\mathrm{i}}, \mathrm{W}_{1}=1-\mathrm{W}_{0}$

$\mu_{1}=\frac{\mu_{0}-\mu_{t}}{1-W_{0}}, \mu_{0}=\frac{\mu_{t}}{W_{0}}, \mu_{t}=\sum_{i=0}^{t} i P_{i}, P_{i}=\frac{n_{i}}{n}$ here $n$ represents total number of pixels and $n_{i}$ represents the number grey level pixels

$\mathrm{n}=\sum_{\mathrm{i}=0}^{\mathrm{L}-1} \quad\left[\mathrm{n}_{\mathrm{i}}\right]$

$P_{i}$, is the probability of occurrence of grey-level $i$.

Class probabilities $\mathrm{w} 0$ and $\mathrm{w} 1$ indicate the portions of the areas occupied by the classes $\mathrm{C} 0$ and $\mathrm{C} 1$. Class means $\mu 0$ and $\mu 1$ serve as estimates of the mean levels of the classes in the original grey-level image. $\eta^{*}$ represents the maximum value of the $\eta$ which is used to measure the separability of classes $\mathrm{C} 0$ and $\mathrm{C} 1$ in the original image or the bimodality of the histogram and it is determined in the range $0 \leq \eta \leq 1$. If the given image has single constant grey level then lower bound (zero) is obtained and when two-valued images are given then upper bound (unity) is obtained.

\subsection{K-means Algorithm}

An unsupervised clustering algorithm that minimize the sum of square distances between all points and the cluster center. It assumes that vector space is formed by the data features and finds a natural clustering in them. The points are clustered around the centriod $\mu_{\mathrm{i}} \forall \mathrm{i}=1,2 \ldots \mathrm{k}$ obtained by minimizing the objectiveV $=\sum_{\mathrm{i}=1}^{\mathrm{k}} \sum_{\mathrm{x}_{\mathrm{j}} \in \mathrm{S}_{\mathrm{i}}}\left(\mathrm{x}_{\mathrm{j}}-\mu_{\mathrm{i}}\right)^{2}$. where $\mathrm{k}$ is the number of clusters.

steps in the algorithm are as follows[20]

1. Compute the intensity distribution (also called the histogram) of the intensities.

2. Initialize the centroids with $\mathrm{k}$ random intensities

3. Repeat the following steps until the cluster a label of the image does not change anymore.

4. Cluster the points based on distance of their intensities from the centroid intensities.

$$
c^{(i)}=\arg \min _{j}\left\|x^{(I)}-\mu_{j}\right\|^{2}
$$

5. now compute the new centroid for each cluster

$$
\mu_{\mathrm{i}}=\frac{\sum_{\mathrm{i}=1}^{\mathrm{m}} 1\left\{\mathrm{c}_{\mathrm{i}}=\mathrm{j}\right\} \mathrm{x}^{(\mathrm{i})}}{\sum_{\mathrm{i}=1}^{\mathrm{m}} 1\left\{\mathrm{c}_{\mathrm{i}}=\mathrm{j}\right\}}
$$

Where $i$ iterates over the all the intensities, $j$ iterates over all the centroids and $\mu_{\mathrm{i}}$ are the centroid intensities.

\subsection{Fuzzy C-means clustering algorithm}

This clustering algorithm introduced by Dunn [21] and later was extended by Bezdek [22]. It is an iterative clustering method that produces an optimal c partition by minimizing the weighted within group sum of squared error objective function JF CM [22]:

$\mathrm{JFCM}=\sum_{\mathrm{K}=1}^{\mathrm{N}} \sum_{\mathrm{I}=1}^{\mathrm{C}}\left(\mathrm{u}_{\mathrm{ik}}\right)^{\mathrm{q}} \mathrm{d}^{2}\left(\mathrm{x}_{\mathrm{k}}, \mathrm{v}_{\mathrm{i}}\right)$

Where $X=\{x 1, x 2, \ldots . . x n\}$ belong to $p$-dimensional vector pace and $\mathrm{n}$ is the number of data items, $\mathrm{c}$ is no of clusters with $2 \leq \mathrm{c} \leq \mathrm{n}$.

A solution of the object function JF CM can be obtained via an iterative process, which is carried out as follows[23].

Initially set values for c, q and $\epsilon$ Initialize the fuzzy partition matrix $U=\left[\mathrm{u}_{\mathrm{ik}}\right]$

Put $b=0$,loop counter

Calculate the clusters centers $c\left\{v_{i}^{(b)}\right\}$ with $U^{(b)}$ :

$$
\mathrm{v}_{\mathrm{i}}^{(\mathrm{b})}=\frac{\sum_{\mathrm{k}=1}^{\mathrm{n}}\left(\mathrm{u}_{\mathrm{ik}}^{(\mathrm{b})}\right)^{\mathrm{q}} \mathrm{x}_{\mathrm{k}}}{\sum_{\mathrm{k}=1}^{\mathrm{n}}\left(\mathrm{u}_{\mathrm{ik}}^{(\mathrm{b})}\right)^{\mathrm{q}}}
$$

Now calculate membership $\mathrm{U}^{(\mathrm{b}+1)}$. for $\mathrm{k}=1$ to $\mathrm{n}$, calculate $\mathrm{I}_{\mathrm{k}}=\left\{\mathrm{i} \mid 1 \leq \mathrm{i} \leq \mathrm{c}, \mathrm{d}_{\mathrm{ik}}=\left\|\mathrm{x}_{\mathrm{k}}-\mathrm{v}_{\mathrm{i}}\right\|=0\right\}$, for kth column of the matrix compute new membership value

$$
\text { if } \mathrm{I}_{\mathrm{k}}=\emptyset \text { then } \mathrm{u}_{\mathrm{ik}}^{(\mathrm{b}+1)}=\frac{1}{\sum_{\mathrm{j}=1}^{\mathrm{c}}\left(\frac{\mathrm{d}_{\mathrm{ik}}}{\mathrm{d}_{\mathrm{jk}}}\right)^{\frac{2}{(\mathrm{q}-1)}}}
$$

else $\mathrm{u}_{\mathrm{ik}}^{(\mathrm{b}+1)}=0 \forall \mathrm{i} \notin \mathrm{I}$ and $\sum_{\mathrm{i} \in \mathrm{I}_{\mathrm{k}}} \mathrm{u}_{\mathrm{ik}}^{(\mathrm{b}+1)}=1$; next k.

If $\left\|\mathrm{U}^{(\mathrm{b})}-\mathrm{U}^{(\mathrm{b}+1)}\right\|<\varepsilon$, stop; otherwise,setb=b+1 and go to step 4.

\subsection{Morphological based method}

Morphology based methods use an algorithm that calculates the connection cost between points in an image - that is, the smallest threshold that will provide a binary path between two points. These methods have been used by $[24,25]$ and it is [25] has used it for the enhancement of the airways in CT slices. The local energy minimization algorithm is used instead of Thresholding the enhanced images. In this method of segmentation erosion and dilation is done, then opening is done which is nothing but smoothing of the image and eliminating the thin protrusions. In other words it is like the erosion followed by an dilation using same structuring element for both operations. closing is to smooth the contours which generally fuses narrow breaks and long thin gulfs, it eliminates small holes and fill the gaps. It is dilation followed by erosion. Thickening and thinning is also morphological operation, In thinning the selected foreground pictures are removed like erosion or opening. Thickening is used to grow selected regions of foreground pixels, similar to dilation or closing. The details of the process is as shown in figure 


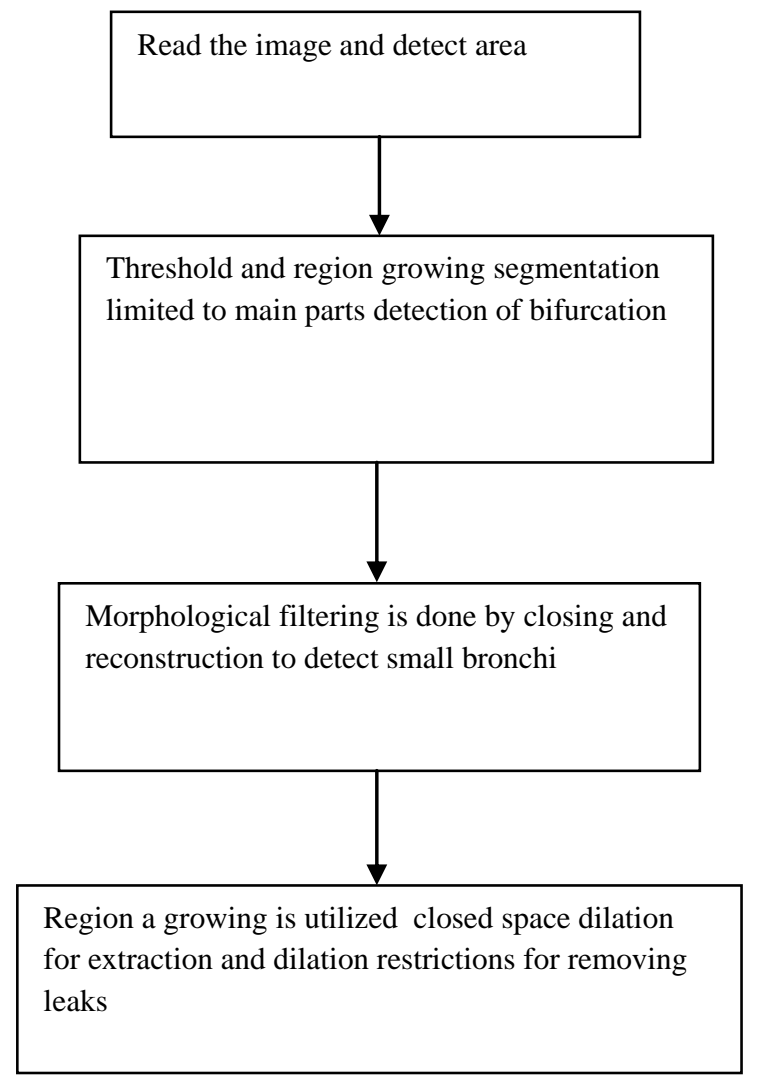

The above figure shows the main theme of implementation. Using the segmented images from the previous as seed points Thresholding and region growing[27] are used on the image, applying progressively from the start. larger branches are segmented using the grayscale Thresholding to avoid the leaks Morphology of binary can be extended to the gray scale

A gray-scale image has $\mathrm{x}$ and $\mathrm{y}$ coordinates of a pixel and the third element is gray-scale value. It can be also applied to the gray-scale structuring element[26]

Gray-scale dilation of $\mathrm{f}$ by $\mathrm{b}$, denoted by $\mathrm{f} \oplus \mathrm{b}$, is defined as

$$
\begin{gathered}
(\mathrm{f} \oplus \mathrm{b})(\mathrm{s}, \mathrm{t})=\max \left(\mathrm{f} f(\mathrm{~s}-\mathrm{x}, \mathrm{t}-\mathrm{y})-\frac{\mathrm{b}(\mathrm{x}, \mathrm{y})}{(\mathrm{s}-\mathrm{x})},(\mathrm{t}-\mathrm{y})\right. \\
\left.\quad \in \mathrm{D}_{\mathrm{f}} ;(\mathrm{s}, \mathrm{y}) \in \mathrm{D}_{\mathrm{b}}\right\}
\end{gathered}
$$

Gray-scale erosion of $\mathrm{f}$ by $\mathrm{b}$, denoted by $\mathrm{f} \Theta \mathrm{b}$, is defined as

$$
\begin{gathered}
(f \ominus b)(s, t)=\max \left\{f(s-x, t+y)-\frac{b(x, y)}{(s+x)},(t+y)\right. \\
\left.\in D_{f} ;(s, y) \in D_{b}\right\}
\end{gathered}
$$

The relation between erosion and dilation is given by

$(\mathrm{f} \ominus \mathrm{b})^{\mathrm{c}}(\mathrm{s}, \mathrm{t})$

$=\left(\mathrm{f}^{\mathrm{c}} \oplus \widehat{\mathrm{b}}\right)(\mathrm{s}, \mathrm{t})$ where $\mathrm{f}^{\mathrm{c}}, \widehat{\mathrm{b}}$ are minimum operators

The opening of a gray image $f$ is defined as

$$
\text { fob }=(\mathrm{f} \ominus \mathrm{b}) \oplus \mathrm{b}
$$

And closing as $\mathrm{f} \bullet \mathrm{b}=(\mathrm{f} \oplus \mathrm{b}) \Theta \mathrm{b}$

The morphological filtering is done and it is defined in [28] and it is used here for the gradient of the image

$$
\operatorname{MG}(\mathrm{f})=\frac{1}{\mathrm{n}} \sum_{\mathrm{i}=1}^{\mathrm{n}}\left[\left(\left(\mathrm{f} \oplus \mathrm{b}_{\mathrm{i}}\right)-\left(\mathrm{f} \ominus \mathrm{b}_{\mathrm{i}}\right)\right) \oplus \mathrm{b}_{\mathrm{i}-1}\right]
$$

After detecting the possible regions using the morphological filtering and reconstruction, bounded space dilation is applied to the binary volume as a region growing technique from the initial seed point. Leaking can be prevented by using the dilation where a restriction is applied to it. The input image is given to the experiment is shown below.

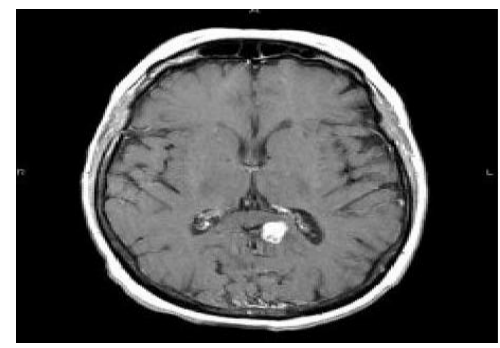

Fig 1: Orginal Image with Varying Background and Foreground Objects

The image is reconstructed with the circular regions removed from it.

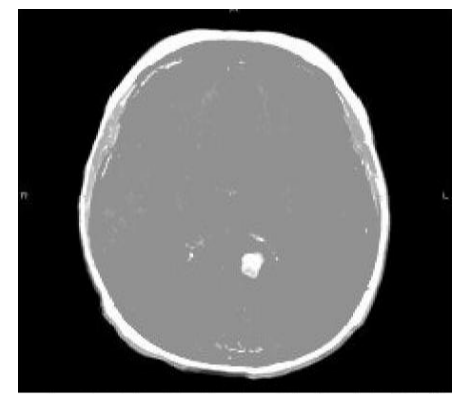

Fig 2: Image with circular images removed

Now we can find the difference between the orginal and the reconstructed image as shown in below figure

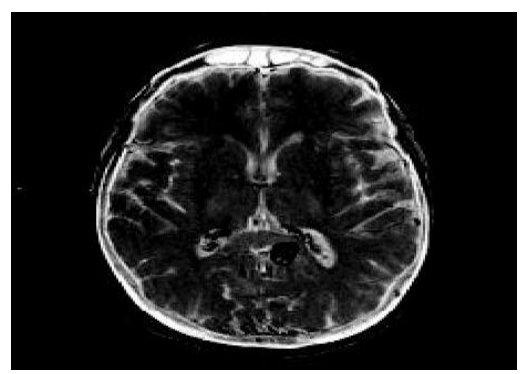

Fig 3 :Image with difference between original and reconstructed

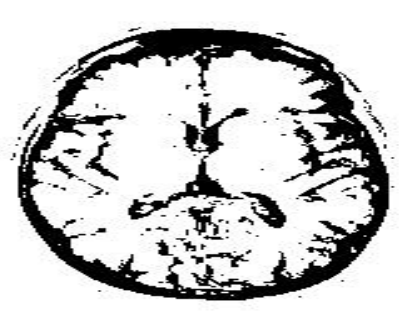

Fig 4: Figure showing the output Image

\section{RESULTS AND DISCUSSIONS}

In this paper segmentation of brain image has been done by using Otsu Thresholding, K-means Clustering method, Fuzzy $\mathrm{C}$-means clustering method and morphological filtering and reconstruction. 
The evaluation of the images is an important concept [29] are done using the Mean Square error, Peak Signal to noise ratio, Normalized Cross-Correlation, Average Difference, Structural Content, Normalized absolute error.

$$
\begin{gathered}
\text { MSE }=\frac{1}{M N} \sum_{j=1}^{M} \sum_{K=1}^{N}\left(x_{j, k}-x_{j, k}^{\prime}\right)^{2} \\
P S N R=10 \log \frac{\left(2^{n}-1\right)^{2}}{M S E}
\end{gathered}
$$

$$
\mathrm{NK}=\frac{\sum_{\mathrm{j}=1}^{\mathrm{M}} \sum_{\mathrm{k}=1}^{\mathrm{N}} \mathrm{x}_{\mathrm{j}, \mathrm{k}} \cdot \mathrm{x}_{\mathrm{j}, \mathrm{k}}^{\prime}}{\sum_{\mathrm{j}=1}^{\mathrm{M}} \sum_{\mathrm{k}=1}^{\mathrm{N}} \mathrm{x}_{\mathrm{j}, \mathrm{k}}^{2}}
$$

\begin{tabular}{|c|c|c|c|c|c|c|c|c|}
\hline Method & Input image & Output image & MSE & PSNR & $\mathrm{NK}$ & $\mathrm{AD}$ & $\mathrm{SC}$ & NAE \\
\hline $\begin{array}{l}\text { Fuzzy C- } \\
\text { Means }\end{array}$ & & & $1.0333 \mathrm{e}+003$ & 17.9885 & 1.0265 & $\begin{array}{l}- \\
9.7043\end{array}$ & 0.9279 & 0.0806 \\
\hline K-Means & & & 414.4834 & 21.9557 & 0.9905 & -0.0743 & 1.0092 & 0.0445 \\
\hline $\begin{array}{l}\text { Morphol } \\
\text { ogical } \\
\text { method }\end{array}$ & & & $1.5154 \mathrm{e}+004$ & 6.3257 & 1.0443 & -63.1118 & 0.6899 & 0.4020 \\
\hline $\begin{array}{l}\text { Otsu } \\
\text { Threshol } \\
\text { ding }\end{array}$ & & & $2.6692 \mathrm{e}+003$ & 13.8670 & 1.0595 & $-\overline{19.1721}$ & 0.8457 & 0.1242 \\
\hline
\end{tabular}

$$
\begin{gathered}
\mathrm{AD}=\frac{\left(\mathrm{x}_{\mathrm{j}, \mathrm{k}}-\mathrm{x}_{\mathrm{j}, \mathrm{k}}^{\prime}\right)}{\mathrm{MN}} \\
\mathrm{SC}=\frac{\sum_{\mathrm{j}=1}^{\mathrm{M}} \sum_{\mathrm{k}=1}^{\mathrm{N}} \mathrm{x}_{\mathrm{j}, \mathrm{k}}^{2}}{\left.\sum_{\mathrm{j}=1}^{\mathrm{M}} \sum_{\mathrm{k}=1}^{\mathrm{N}} \mathrm{x}_{\mathrm{j}, \mathrm{k}}^{\prime}\right)^{2}} \\
\mathrm{NAE}=\frac{\sum_{\mathrm{j}=1}^{\mathrm{M}} \sum_{\mathrm{k}=1}^{\mathrm{N}}\left|\mathrm{x}_{\mathrm{j}, \mathrm{k}}-\mathrm{x}_{\mathrm{j}, \mathrm{k}}^{\prime}\right|}{\sum_{\mathrm{j}=1}^{\mathrm{M}} \sum_{\mathrm{k}=1}^{\mathrm{N}}\left|\mathrm{x}_{\mathrm{j}, \mathrm{k}}\right|}
\end{gathered}
$$

Table 1. Table showing the Summary of Results

\section{CONCLUSION}

An medical MRI brain is given as input to the experiment and segmentation is done using the Otsu Thresholding, K-means Clustering method, Fuzzy C-means clustering method and morphological filtering and reconstruction. The performance

of these algorithms are measured using the Mean Square error, Peak Signal to noise ratio, Normalized CrossCorrelation, Average Difference ,Structural Content, Normalized absolute error. The computational time taken by $\mathrm{K}$-means is more than Fuzzy C-Means, making FCM better in the clustering technique. The performance of the Otsu Thresholding is better than other Thresholding techniques and also the clustering techniques. Morphological opening and closing is better in the showing of the content of the image than the other techniques.

\section{REFERENCES}

[1] Sujata saini and Komal Arora "Study Analysis on the different Image segmentation Techniques" international journal of information and computation ISSN 09742239 volume 4, Number 14(2014), pp 1445-1452.

[2] Image segmentation technique Rajeshwar Dass, Priyanka, Swapna Devi IJECT vol 3,Issue 1,Jan-March 2012.

[3] Edge Detection techniques evaluation and comparisions Ehsan applied mathematical sciences vol 2, 2008 no 31, $1507-1520$

[4] Rafael C Ganzalez,Richard E Woods, "Digital Image processing",2nd ed ,Beijing Publishing Housing of Electronics Industry,2007.

[5] S.M. Larie and S.S. Abukmeil. Brain abnormality in schizophrenia: a systematic and quantitative review of volumetric magnetic resonance imaging studies. J. Psych., 172:110-120, 1998. 
[6] P. Taylor. Invited review: computer aids for decisionmaking in diagnostic radiology - a literature review. Brit. J. Radiol.., 68:945-957, 1995.

[7] A.P. Zijdenbos and B.M. Dawant. Brain segmentation and white matter lesion detection in MR images. Critical Reviews in Biomedical Engineering, 22:401-465, 1994.

[8] A.J. Worth, N. Makris, V.S. Caviness, and D.N. Kennedy. Neuroanatomical segmentation in MRI: technological objectives. Int. J. Patt. Rec. Art. Intel., 11:1161-1187, 1997.

[9] V.S. Khoo, D.P. Dearnaley, D.J. Finnigan, A. Padhani, S.F. Tanner, and M.O. Leach. Magnetic resonance imaging (MRI): considerations and applications in radiotheraphy treatment planning. Radiother. Oncol., 42:1-15, 1997.

[10] H.W. Muller-Gartner, J.M. Links, et al. Measurement of radiotracer concentration in brain gray matter using positron emission tomography: MRI-based correction for partial volume effects. J. Cereb. Blood Flow Metab., 12:571-583, 1992

[11] N. Ayache, P. Cinquin, I. Cohen, L. Cohen, F. Leitner, and O. Monga. Segmentation of complex threedimensional medical objects: a challenge and a requirement for computer-assisted surgery planning and performance. In R.H. Taylor, S. Lavallee, G.C. Burdea, and R. Mosges, editors, Computerintegrated surgery: technology and clinical applications, pages 59-74. MIT Press, 1996.

[12] W.E.L. Grimson, G.J. Ettinger, T. Kapur, M.E. Leventon, W.M.Wells, et al. Utilizing segmented MRI data in image-guided surgery. Int. J. Patt. Rec. Art. Intel., 11:1367-1397, 1997.

[13] Prof. Dinesh D. Patil, Ms. Sonal G. Deore, "Medical Image Segmentation: A Review", IJCSMC, Vol. 2, Issue 1, January 2013, pg.22 - 27.

[14] Hu A, Grossberg B, Mageras C. Survey of recent volumetric medical image segmentation techniques. Biomedical Engineering. 2009;321-346. http://www.intechopen.com/source/pdfs/8807/InTechSurvey_of_recent_volumetric_medical_image_segmenta tion_techniques.pdf

[15] Zuva T, Olugbara OO, Ojo SO et al. Image segmentation, available techniques, developments and open issues. Canadian Journal on Image Processing and Computer Vision 2011;2(3):20-29. http://www.ampublisher.com/Mar\%202011/IPCV-1103011-Image-Segmentation-Available-TechniquesDevelopments-Open-Issues.pdf

[16] Pham DL, Xu C, Prince JL. A survey of current methods in medical image segmentation. Annual Review of Biomedical Engineering, 2000;2:315-338. http://www.tecn.upf.es/ afrangi/ibi/CurrentMethodsInIm ageSegmentation_Phan2000.pdf
[17] Ng, H.F., 2006. Automatic thresholding for defect detection. Pattern Recognition Lett. 27 (14), 1644-1649.

[18] Otsu, N., "A Threshold Selection Method from GrayLevel Histograms," IEEE Transactions on Systems, Man, and Cybernetics, Vol. 9, No. 1, 1979, pp. 62-66.

[19] Ch. Hima Bindu and K. Satya Prasad "An Efficient Medical Image Segmentation Using Conventional OTSU Method" International Journal of Advanced Science and Technology Vol. 38, January, 2012

[20] Suman Tatiraju and Avi Mehta "Image Segmentation using k-means clustering, EM and Normalized Cuts" www.ics.uci.edu/ dramanan/teaching/ics273a.../avim_re port.pdf

[21] Dunn, J. C.: A Fuzzy Relative of the ISODATA Process and its Use in Detecting Compact Well Separated Clusters. Journal of Cybernetics, Vol. 3, 1974, pp. 3257.

[22] Bezdek, J. C.: Pattern Recognition with Fuzzy Objective Function Algorithms. New York: Plenum Press, 1981.

[23] Yong Yang and Shuying Huang "IMAGE SEGMENTATION BY FUZZY C-MEANS CLUSTERING ALGORITHM WITH A NOVEL PENALTY TERM" Computing and Informatics, Vol. $26,2007,17-31$.

[24] Pr^eteux, F., Fetita, C., Capderou, A., Grenier, P.: Modeling, segmentation, and caliber estimation of bronchi in high resolution computerized tomography. Journal of Electronic Imaging 8 (1999) 36-45.

[25] Fetita, C., Preteux, F., Beigelman-Aubry, C., Grenier, P. Pulmonary Airways: 3-D Reconstruction From Multislice CT and Clinical Investigation. IEEE Transactions on Medical Imaging 23 (2004) 1353-1364

[26] K. Parvati, B. S. Prakasa Rao, and M. Mariya Das "Image Segmentation Using Gray-Scale Morphology and Marker-Controlled Watershed Transformation" Hindawi Publishing Corporation Discrete Dynamics in Nature and Society Volume 2008, Article ID 384346, 8 pages doi: $10.1155 / 2008 / 384346$

[27] Benjamin Irving, Paul Taylor, and Andrew ToddPokropek "3D segmentation of the airway tree using a morphology based method" EXACT'09-297

[28] L. Vincent and P. Soille, "Watersheds in digital spaces: an efficient algorithm based on immersion simulations," IEEE Transactions on Pattern Analysis and Machine Intelligence, vol. 13, no. 6, pp. 583-598,1991.

[29] Kalpana Shrivastava, Neelesh Gupta and Neetu Sharma " Medical Image Segmentation using Modified K Means Clustering" International Journal of Computer Applications (0975 - 8887) Volume 103 - No 16, October 2014 